\title{
Intuitively detecting what is hidden within a visual mask: Familiar-novel discrimination and threat detection for unidentified stimuli
}

\author{
Anne M. Cleary • Anthony J. Ryals • Jason S. Nomi
}

Published online: 20 April 2013

(C) Psychonomic Society, Inc. 2013

\begin{abstract}
Recognition without identification is the finding that, among recognition test items that go unidentified (as when a word is unidentified from a fragment), participants can discriminate those that were studied from those that were unstudied. In the present study, we extended this phenomenon to the more life-like situation of discriminating known from novel stimuli. Pictures of famous and nonfamous faces (Exp. 1), famous and nonfamous scenes (Exp. 2), and threatening and nonthreatening images (Exp. 3) were filtered in order to impede identification. As in list-learning recognition-without-identification paradigms, participants attempted to identify each image (e.g., whose face it was, what scene it was, or what was in the picture) and rated how familiar the image seemed on a scale of 0 (very unfamiliar) to 10 (very familiar). Among the unidentified stimuli, higher familiarity ratings were given to famous than to nonfamous faces (Exp. 1) and scenes (Exp. 2), and to threatening than to nonthreatening living/animate (but not to nonliving/nonanimate) images (Exp. 3). These findings suggest that even when a stimulus is too occluded to allow for conscious identification, enough information can be processed to allow a sense of familiarity or novelty with it, which appears also to be related to the sense of whether or not a living creature is a threat. That the sense of familiarity for unidentified stimuli may be related to threat detection for living or animate things suggests that it may be an adaptive aspect of human memory.
\end{abstract}

Keywords Familiarity $\cdot$ Recognition memory $\cdot$ Recognition without identification $\cdot$ Threat detection

A. M. Cleary $(\bowtie) \cdot$ A. J. Ryals $\cdot$ J. S. Nomi

Department of Psychology, Colorado State University,

1976 Campus Delivery,

Fort Collins, CO 80523-1876, USA

e-mail: Anne.Cleary@colostate.edu
When Federico Zeri and Evelyn Harrison and Thomas Hoving and Georgios Dontas-and all the others-looked at the kouros and felt an "intuitive repulsion," they were absolutely right. In the first two seconds of looking - in a single glance - they were able to understand more about the essence of the statue than the team at the Getty was able to understand after fourteen months.

\section{-Malcolm Gladwell, Blink}

In the book The Girl With the Dragon Tattoo, Stieg Larsson (2008) writes about a gut feeling that the main character keeps having upon studying a picture album. The character, Blomkvist, cannot identify what in the set of pictures is causing the feeling, but the feeling seems to alert him to the fact that there is something of interest there, something that may help him to solve the case on which he is working (something that he may identify if he keeps trying). Larsson writes, "He felt a fresh excitement, and over the years Blomkvist had learned to trust his instincts. These instincts were reacting to something in the album, but he could not yet say what it was . . . After Berger's visit in May, he had studied the album again, sitting for three hours, looking at one photograph after another, as he tried to rediscover what it was that he had reacted to" (p. $224 \&$ p. 235).

This type of experience - reacting to something with a gut feeling or "instinct" about what was just seen - is reminiscent of the type of decision-making illustrated in the quote above from Malcolm Gladwell's (2005) book Blink, and it may reflect an adaptive memory process: It presents a means of making a rapid decision in the face of minimal information that could at times be life-saving (e.g., in combat or SWAT team operations, one may have to make an urgent decision when a quick glance or rapid movement is 
all that one has to go on; in other situations, one may have to make a decision about how to interact with a person when a feeling of familiarity is all that one has to go on). These examples relate to survival in ways that may be relevant to survival in our ancestral past, in which case, it is likely that the cognitive processes that produce these types of gut feelings were shaped by evolution. In the present study, we attempt to relate these types of real-life intuitive feelings about situations to a laboratory phenomenon known as recognition without identification, and also explores the idea that such feelings, at least in some cases, may have been shaped by evolution.

Recognition without identification (RWI) is the finding that participants in laboratory situations can discriminate between studied and nonstudied items when the items themselves cannot be identified (Cleary, 2011; Cleary \& Greene, 2000, 2001, 2005; Cleary, Langley, \& Seiler, 2004; Cleary \& Reyes, 2009; Cleary \& Specker, 2007). In this method, the identification of recognition test items is hindered, often by perceptually degrading the stimuli, such as by fragmenting the test items (e.g., Cleary \& Greene, 2000, 2001; Cleary et al., 2004; Cleary, Winfield, \& Kostic, 2007; Kostic \& Cleary, 2009; Peynircioğlu, 1990) or by rapidly presenting and masking them (e.g., Arndt, Lee, \& Flora, 2008; Cleary \& Greene, 2005; Langley, Cleary, Woods, \& Kostic, 2008; Morris, Cleary, \& Still, 2008). It has been suggested that the RWI effect results from the fact that people can detect an item's relative familiarity when the available stimulus information is too minimal to allow conscious identification (e.g., Arndt et al., 2008; Cleary, 2008; Cleary \& Greene, 2001, 2005).

Though the RWI method follows from a long tradition of studying recognition memory using list-learning paradigms, it may relate to the real-life experience of having a gut feeling or intuition about a situation, as in the examples provided at the start of this introduction. For example, if RWI indeed results from an ability to detect an unidentified item's relative familiarity within the context of an experiment, it may reflect a more general ability to detect familiarity in real life, such as when recognizing a face as familiar without being able to identify who the person is or how the person is known. In this type of real-life familiarity detection, a person is recognizing that something is known as opposed to being novel, whereas with the laboratory-based RWI phenomenon, a person is discriminating recently from nonrecently encountered items. In discussing the "mere-recognition" basis of the recognition heuristic, whereby people may separate the previously experienced from the novel in day-to-day life on the basis only of a sense of familiarity, Goldstein and Gigerenzer (2002) noted this critical difference between real-life familiarity and familiarity in list-learning paradigms, stating, "This use of the term [recognition] needs to be distinguished from another use, which might be characterized as 'recognition of items familiar from a list' " (p. 77). Extending laboratory studies of RWI to the more life-like experience of discriminating the familiar from the novel is important because this will aid in determining how people can use minimal information to make decisions in real-world contexts, particularly when not enough information is available to allow for conscious identification of the situation.

Bolte and Goschke (2008) carried out a study that may be the closest yet to demonstrating the laboratory-based RWI phenomenon in a more life-like context of discriminating the known from the novel. They presented participants with fragments of objects, some of which were "coherent," in that they were merely fragmented versions of the original drawings (e.g., a typewriter); some were "incoherent," in that the same fragment pieces from the "coherent" condition were scrambled. When participants could not identify the fragmented objects, they could still discriminate between coherent and incoherent fragments. That is, they could recognize fragments as coming from "known" objects (i.e., typewriter) without being able to identify those objects. Bolte and Goschke suggested that activation of existing knowledge representations can exert an effect on people's decisions, even when the knowledge driving the decisions could not itself be consciously accessed; they referred to this ability as "intuition" (an ability to make decisions without being able to verbalize their basis). It could also be viewed as a gut feeling or sense, an idea that is compatible with the notion of familiarity as a mere feeling.

The results of Bolte and Goschke (2008) suggest that RWI may extend to real-life situations. That is, when known and novel stimuli are unidentifiable, people may be able to discriminate between the known and the novel on the basis of only a feeling or sense about those stimuli, and that feeling or sense might be familiarity. In the present study, we investigated this idea further, with the goal of linking the established laboratory RWI phenomenon with the more life-like task of discriminating the known from the novel. If the RWI effect can be extended to paradigms in which the discrimination to be made is between known and novel stimuli, rather than between studied and nonstudied stimuli, this may present a means of better understanding the process that underlies rapid, intuition-based decisions.

In Experiment 1, we investigated whether participants could discriminate between unidentified faces of famous and nonfamous people when the faces were occluded with a filter mask to make them unidentifiable. In Experiment 2, we extended Experiment 1's paradigm to scenes to determine whether participants could discriminate between unidentified famous and nonfamous scenes or locations. 
Hereafter, we will refer to such discrimination as "realworld RWI."

The present study also investigated the idea that RWI in situations of discriminating the known from the novel (i.e., real-world RWI) may have been shaped by evolution (i.e., may be adaptive). There is reason to suspect that such an ability would be adaptive: It allows people a basis on which to make decisions when faced with incomplete or partial information, or even when so little information is available that no aspect of the situation itself can be consciously identified. If nothing else, an intuitive sense about a situation can alert a person to the fact that something familiar may be present that should cause him or her to give pause before proceeding, or that something may be worth investigating further, even if what that something is currently unknown, as in the example from Stieg Larsson's novel in the first paragraph above. Such an ability might lead to survival benefits in high-stakes situations, and thus might have been shaped by evolution.

To investigate the hypothesis that the ability to discriminate known from novel unidentified stimuli (i.e., real-world RWI) is adaptive, in Experiment 3 we examined whether participants could discriminate, via familiarity ratings, between unidentified threatening images and unidentified nonthreatening images when the images were occluded with a filter mask to make them unidentifiable. We were specifically interested in whether unidentified threatening stimuli would lead to higher familiarity ratings than would unidentified nonthreatening stimuli.

In terms of the potential adaptiveness of any such ability, prior research suggested that with supraliminal stimuli, threats can be rapidly detected from images that contain only low-spatial-frequency, coarse information (Mermillod, Droit-Volet, Devaux, Schaefer, \& Vermeulen, 2010). In fact, the high-spatial-frequency advantage that is usually found for supraliminal stimuli disappeared when threat stimuli were used, suggesting that people may be able to recognize threats with less perceptual information than other types of stimuli. This threat advantage to processing was limited to living (i.e., animate) threats; it was not found with nonliving (i.e., inanimate) threats. This finding may relate to other findings on adaptive cognitive processes, such as evidence that attentional cognitive-processing advantages are associated with animate objects (i.e., animals) relative to inanimate objects, and that this advantage may reflect "ancestral priorities" (New, Cosmides, \& Tooby, 2007). More recently, animacy has been shown to influence memory, such that animate stimuli are remembered better than inanimate stimuli (VanArsdall, Nairne, Pandeirada, \& Blunt, in press). Given this abundance of evidence suggesting that something is special about living/animate stimuli with regard to cognitive processing, in our Experiment 3, we examined whether it makes a difference to the real-world RWI effect whether the threatening stimuli are living (i.e., animate) or nonliving (i.e., inanimate). If a difference were to emerge, such that unidentified living threats produce a greater sense of familiarity than do either unidentified living nonthreats or unidentified nonliving threats, this would provide some support for the idea that intuition-based familiarity detection may be an adaptive process that was shaped by evolution.

\section{Experiment 1}

In Experiment 1, we examined whether participants could discriminate, via familiarity ratings, between famous faces and nonfamous faces when the faces could not be identified because of a visual noise filter mask.

\section{Method}

Participants A group of 22 Colorado State University students participated in exchange for course credit.

Materials The stimuli were 120 faces, 60 of which were head shots of famous faces (30 male and 30 female celebrities), and 60 of which were head shots of nonfamous faces selected from nonfamous actors and models whose head shots were published on the Web ( 30 male and 30 female). As an independent index of the famousness versus nonfamousness of these two different pools of faces, 29 additional participants were presented with the 120 faces and asked to rate how famous each was, using a scale of 1 (not at all famous) to 9 (very famous). The ratings of famousness were indeed significantly higher for faces that came from our pool of selected famous faces $(M=7.63, S D=$ $0.76)$ than for faces that came from our pool of selected nonfamous faces $(M=2.87, S D=0.90), t(28)=20.82, S E=$ $0.23, p<.001$, Cohen's $d=3.94$. The pictures of faces were each $175 \times 175$ pixels in size. The noise mask for impeding face identification was created by running each face picture through a Gaussian monochromatic noise filter of $150 \%$ in Photoshop. This noise filter applied random pixels to the picture using a Gaussian distribution, such that the color values used for the noise creation were distributed on a bellshaped (i.e., Gaussian) curve. Choosing the monochromatic version of the filter allowed for some degree of color preservation within the image, because the filter applied pixels that differed only in tone, and not in actual color, from the original image (see Fig. 1A).

Procedure Participants were presented with the 120 filtered images in a randomized order. For each filtered face presented, participants were first asked to try to identify the person by typing in the person's name or any identifying 


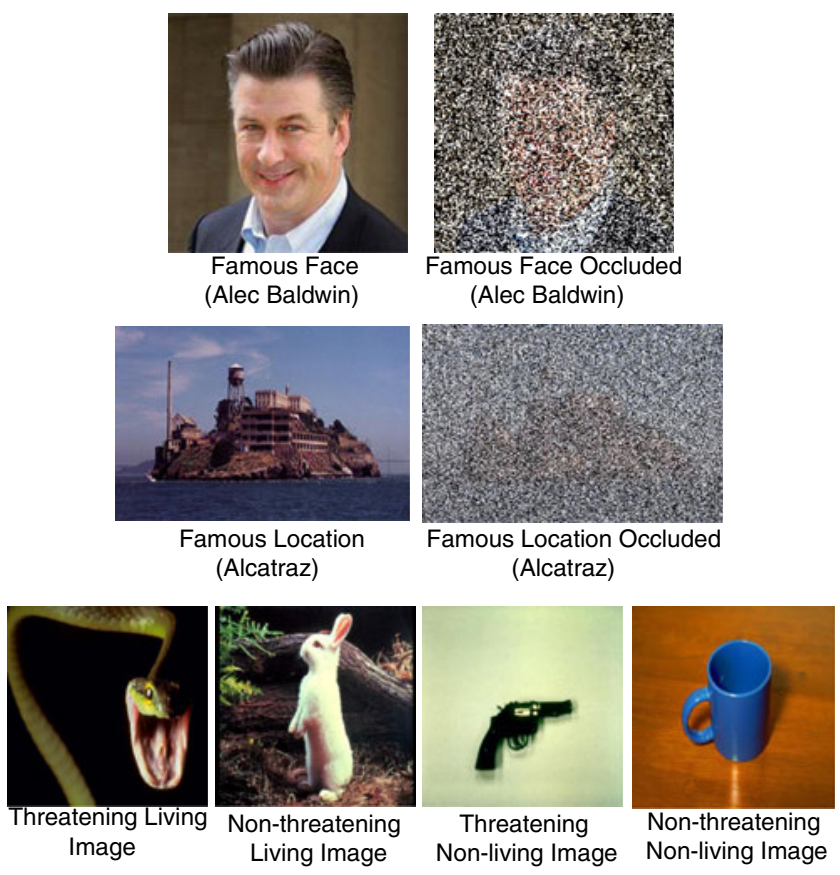

Fig. 1 (A) Example face stimulus from Experiment 1 before and after a $150 \%$ monochromatic noise filter. (B) Example scene stimulus from Experiment 2 before and after a $200 \%$ monochromatic noise filter. (C) Examples of threatening and nonthreatening stimuli, living on the left and nonliving on the right, from Experiment 3. These stimuli were run through a $150 \%$ monochromatic noise filter, as in Experiment 1

information about the person (such as a movie in which the person had acted). Then, regardless of whether the face or any correct information about it could be identified, the participant was prompted to rate how familiar the face seemed using a scale of 0 (very unfamiliar) to 10 (very familiar). Once all 120 trials were completed, the participant was then presented with the pictures of the 60 famous faces, unfiltered, in a random order. For each unfiltered famous face, the participant was asked to identify the person by typing in his or her name. The participant was also encouraged to type in any known semantic information about the person, such as a movie that the person had played in or a character that was played by the person. These data were used to conditionalize the familiarity ratings given to the unidentified filtered famous faces from earlier in the experiment, in terms of whether the faces would have been identifiable had they not been filtered/occluded. In this way, we were able to ensure that participants were not simply recognizing a celebrity face through the filter as a famous person, without ever having known the person's name or identifying information. We only considered data from celebrities that would have been identifiable if presented without the filter. We considered celebrities who were correctly named when unfiltered as being identifiable, as well as celebrities for whom correct identifying information (e.g., a movie or TV show in which the person acted, the name of a character that the person played, etc.) was indicated. For instance, if a participant was unable to identify a famous face as "Alec Baldwin," but indicated that the face belonged to "the actor from 30 Rock," this was binned as an identified face.

\section{Results}

The visual noise filter was successful at hindering identification of the faces. On average, participants identified fewer than $1 \%$ of the filtered famous faces (only 0.59 out of 60 faces, on average), including those instances in which participants indicated correct information about the person but not the person's name. Considering that an average of $56 \%$ of the famous faces were identified when the faces were later presented unfiltered ( 33.82 out of 60 , on average), the filter appears to have been successful at impeding identification of the faces. As is shown in Fig. 2, among filtered face images that went unidentified, participants gave higher familiarity ratings to those that contained famous filtered faces than to those that contained nonfamous filtered faces, $t(21)=2.40, S E=0.11, p<.05$, Cohen's $d=0.52$, even though we only considered ratings given to famous faces that would have been identifiable if not filtered. The familiarity ratings given to unidentified filtered famous faces that were not later identifiable when unfiltered $(M=2.63, S D=1.56)$ were also significantly higher than the ratings given to nonfamous filtered faces $(M=2.30, S D=1.33), t(21)=2.33, S E=0.14$, $p<.05$, Cohen's $d=0.51$, and did not differ significantly from the ratings given to famous faces that were later identifiable when unfiltered $(M=2.58, S D=1.25), t(21)=$ 0.39 . Although the mean familiarity ratings were comparable for unidentified famous faces that were versus were not later identified when unfiltered, our main focus was on the former, because those faces, if "fully seen" through the noise, would also have been namable (given that they were namable when seen without noise). Among the faces unnamed when unfiltered, we could not be certain which might have been "fully seen" when filtered but could not be indicated as such because their names were not known (as participants were unable to name these faces when they were unfiltered).

\section{Experiment 2}

Another important discrimination that people may need to make in day-to-day life is between familiar and novel scenes, as a quick glance of a scene may indicate whether everything is as expected (i.e., familiar), even if only minimal information about the scene is available. In Experiment 2, we extended Experiment 1's paradigm to scenes to determine whether participants can discriminate between unidentified famous and nonfamous scenes. 
Fig. 2 Familiarity ratings given to unidentified images. Error bars represent withinsubjects confidence intervals (Loftus \& Masson, 1994). (A) Familiar-novel discrimination among unidentified faces in Experiment 1. (B) Familiar-novel discrimination among unidentified scenes in Experiment 2. (C) In Experiment 3, discrimination between threatening and nonthreatening unidentified images occurred only among images of living things; such discrimination did not occur among nonliving things

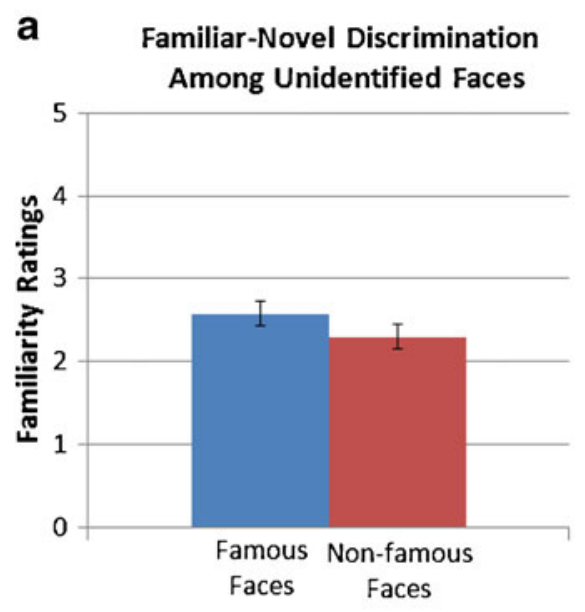

b Familiar-Novel Discrimination Among Unidentified Scenes

c Threat/Non-threat Discrimination Among Unidentified Images

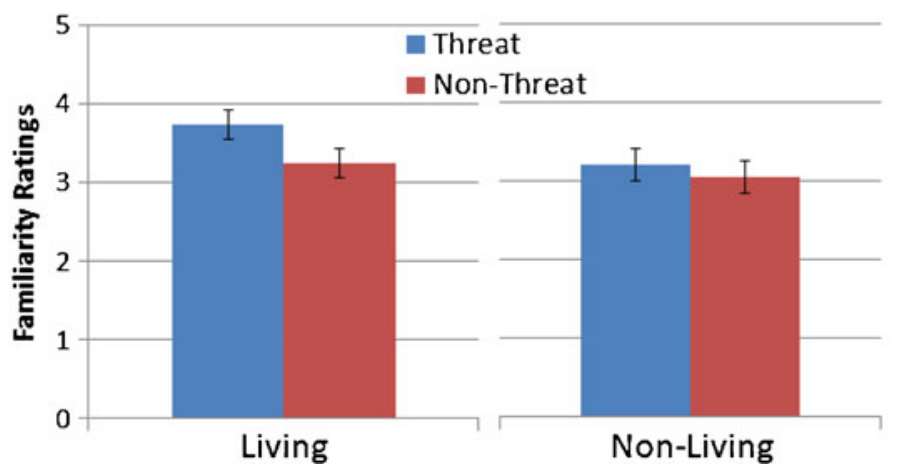

\section{Method}

Participants A group of 48 Colorado State University students participated in exchange for course credit. One was excluded for not identifying any of the scenes when they were later presented unoccluded.

Materials The stimuli were created in the same way as those from Experiment 1, with the exception that, rather than faces, famous and nonfamous scenes and locations were used, and the nonfamous scenes were each chosen to be similar in appearance to one of the famous scenes. For example, for the famous scene Alcatraz, the similar nonfamous scene was a photograph of an anthill in a desert landscape. Both famous and nonfamous scene images were obtained via Web photograph searches. As in Experiment 1, to independently assess the famousness versus nonfamousness of the scenes that we used, we had 29 additional participants rate the famousness of the 120 scenes using a scale of 1 (not at all famous) to 9 (very famous). The ratings of famousness were significantly higher for scenes that came from our pool of selected famous scenes $(M=7.00, S D=1.03)$ than for scenes that came from our pool of selected nonfamous scenes $(M=3.48, S D=0.87), t(28)=22.31, S E=0.16, p<.001$, Cohen's $d=4.22$. Scene images ranged from $300 \times 300$ to
$400 \times 400$ pixels and, because the pilot data suggested that the scenes were more identifiable than the faces from a Gaussian monochromatic noise filter of $150 \%$ (the filter that had been used in Exp. 1), we instead used a Gaussian monochromatic noise filter of $200 \%$ for the scenes.

Procedure The procedure was the same as in Experiment 1, except that the questions now pertained to scenes rather than faces.

\section{Results}

Though participants were better at identifying the filtered scenes than they were at identifying the filtered faces used in Experiment 1, the filter was still successful at hindering identification of the scenes. An average of $8.47(14 \%)$ of the filtered scenes were identified, despite the fact that, when the scenes were presented unfiltered at the end of the experiment, an average of $45 \%$ were identified $(26.98$ out of 60 on average). As can be seen in Fig. 2, among the filtered scenes that went unidentified, participants gave higher familiarity ratings to those that were famous than to those that were nonfamous, $t(46)=5.53, S E=0.11, p<.001$, Cohen's $d=0.82$, even though we only considered ratings given to famous scenes that would have been identifiable if 
not filtered. The familiarity ratings given to unidentified filtered famous scenes that were not later identifiable when unfiltered $(M=4.20, S D=1.36)$ were also significantly higher than the ratings given to nonfamous filtered scenes $(M=3.36, S D=1.24), t(46)=7.31, S E=0.12, p<.001$, Cohen's $d=1.08$, and were also higher than the ratings given to famous filtered scenes that were later identifiable when unfiltered $(M=3.97, S D=1.49), t(46)=2.00, S E=0.12$, $p=.05$, Cohen's $d=0.29$. Although the mean familiarity ratings were higher for unidentified famous scenes that were not later identified when unfiltered than for those that were later identified when unfiltered (note that we classified unfiltered scenes as being identified if any identifying information about the scene had been indicated), our main focus was on the latter, because those scenes, if "fully seen" through the noise, would also have been namable (given that they were namable when later seen without noise). In the former condition, we could not be certain which scenes might have actually been "fully seen" but could not be indicated as such, because their names were not known (as participants were unable to name these scenes when they were unfiltered). In fact, the reason why the ratings were highest in this condition may have been because some of the scenes were, in fact, "fully seen"- - that is, recognized as having been seen before - but the name was simply not known.

\section{Experiment 3}

Additional real-world stimuli that are important to be able to detect in the face of minimal information are those that are threatening. In Experiment 3, we examined whether unidentifiable threatening images would seem more familiar than unidentifiable nonthreatening images. In addition, because some research has suggested that living threats may be more detectable as threats than nonliving threats among difficult-to-identify filtered stimuli (Mermillod et al., 2010), we also examined whether any observed tendency to rate unidentifiable threatening images as being more familiar than unidentifiable nonthreatening images would differ for living (i.e., animate) versus nonliving (i.e., inanimate) stimuli. On the basis of prior research, and of our assumption that increased threat detection for minimally processed living things might manifest in the form of an intuitive sense, we hypothesized that the sense of familiarity might be greater for unidentified living than for unidentified nonliving threats.

A further benefit to completing Experiment 3 was that the stimuli were conducive to comparing familiarity ratings between the threatening and nonthreatening stimuli at comparable levels of identifiability of the stimuli through the noise masks. Whereas the novel (nonfamous) stimuli from Experiments 1 and 2 were not inherently identifiable in a way that would allow us to compare the famous and nonfamous images across categories of comparable identifiability (e.g., a nonfamous face had no associated name to use to indicate an identification of the face), the stimuli in Experiment 3 were all inherently identifiable (e.g., a gun, a coffee mug, a snake, or a bunny), and thus, we could make such a comparison across all of the familiarity rating categories to determine whether the familiarity ratings for the threatening versus nonthreatening stimuli varied according to the likelihood of identification. Toward this end, in Experiment 3, we also examined familiarity ratings for unidentified images as a function of the relative identifiability of the images across participants.

\section{Method}

Participants A group of 68 Colorado State University students participated in exchange for course credit. Eight were excluded for failure to attempt to identify the images.

Materials The stimuli were created in the same way as those in Experiments 1 and 2, with the exception that, rather than faces or scenes, pictures of threatening and nonthreatening images, half living and half nonliving from each category, were used (see Fig. 1). The majority were taken from the International Affective Picture System (IAPS; Lang, Bradley, \& Cuthbert, 2005); however, in order to obtain a large enough sample and equal numbers of items per category, eight living and five nonliving threat images were additionally chosen from the Internet to be comparable to those taken from Lang et al. Eighty pictures altogether were used, with 20 in each of the four categories (living threat, nonliving threat, living nonthreat, or nonliving nonthreat). Note that the living stimuli were animate and nonliving stimuli were inanimate. The images were all $350 \times 350$ pixels, and a monochromatic noise filter of $150 \%$ was used.

To independently verify that our threatening stimuli were indeed more threatening than our nonthreatening stimuli, we had 16 additional participants rate how threatening the 80 scenes seemed to them, using a scale of 1 (not at all threatening) to 9 (very threatening). A $2 \times 2$ Threat Status (threatening vs. nonthreatening) $\times$ Living Status (living vs nonliving) repeated measures ANOVA revealed a main effect of threat status, such that threat ratings were significantly higher for images that came from our pool of selected threatening images for both living $(M=6.46, S D=1.46)$ and nonliving ( $M=7.08, S D=1.09)$ images than for images that came from our pool of selected nonthreatening images for both living $(M=2.07, S D=0.88)$ and nonliving $(M=1.95$, $S D=0.67)$ images, $F(1,15)=444.06, M S E=0.82, p<.001$, $\eta_{\mathrm{p}}{ }^{2}=.97$. There was no main effect of living status, $F(1,15)=2.40, M S E=0.42, p=.14, \eta_{\mathrm{p}}{ }^{2}=.14$. Interestingly, however, the interaction was significant, $F(1,15)=8.53$, 
$M S E=0.25, p<.05, \eta_{\mathrm{p}}{ }^{2}=.36$, such that the difference between the ratings for threatening and nonthreatening images was greater for nonliving than for living stimuli. Indeed, a paired-samples $t$ test performed on the ratings given only to our pool of threatening images revealed that threat ratings were significantly higher for nonliving than for living threatening images, $t(15)=2.36, S E=0.26, p<.05, d=0.61$. This is interesting, in light of prior research from the same pool of stimuli suggesting that perceptually degraded living threats are more detectable among threatening stimuli than among nonliving threats (e.g., Mermillod et al., 2010). This prior research had led to our hypothesis that unidentifiable living threats would lead to higher familiarity ratings than would unidentifiable nonliving threats. Our finding that threat ratings among unmasked images were actually higher for nonliving than for living threatening stimuli suggests that at a conscious level, our nonliving threatening stimuli were perceived as being more threatening than our living threatening stimuli. Therefore, if we indeed were to obtain higher familiarity ratings for unidentified living than for unidentified nonliving threatening stimuli, it would suggest that there may be a difference between the processes underlying conscious threat identification and the possibly more intuition-based sense of familiarity that occurs with unidentified threatening images occluded by means of a visual noise mask.

Procedure The procedure was the same as in Experiments 1 and 2 .

\section{Results}

The visual noise filter was successful at hindering identification of the images. Among the filtered images, participants identified an average of $32 \%$, despite the fact that when the stimuli were presented unfiltered at the end of the experiment, an average of $95 \%$ were identified.

Our primary interest was in the ratings given to filtered scenes that went unidentified. Among these, a $2 \times 2$ Threat Status (threatening vs. nonthreatening) $\times$ Living Status (living vs. nonliving) repeated measures ANOVA revealed main effects of both threat status, $F(1,59)=8.88, M S E=$ $0.71, p<.01, \eta_{\mathrm{p}}{ }^{2}=.13$, and living status, $F(1,59)=24.87$, $M S E=0.33, p<.001, \eta_{\mathrm{p}}{ }^{2}=.30$ (see Fig. 2). However, these main effects were qualified by an interaction, $F(1,59)=$ $3.85, M S E=0.43, p=.05, \eta_{\mathrm{p}}{ }^{2}=.06$, that suggests that the tendency to give higher familiarity ratings to threat than to nonthreat stimuli was greater for living than for nonliving things, and that both main effects were carried largely by the tendency for ratings to be highest in the living threat condition. Follow-up $t$ tests revealed that the ability to discriminate threatening from nonthreatening stimuli that went unidentified was indeed limited to the category of living things, $t(59)=3.79, S E=.13, p<.001, d=0.49$, as the difference in familiarity ratings was not significant for nonliving things, $t(59)=1.08, S E=0.15, p=.28$. Interestingly, participants also gave higher familiarity ratings to unidentified living threats than to unidentified nonliving threats, $t(59)=$ $4.31, S E=0.12, p<.001, d=0.56$. Thus, the highest familiarity ratings were given to living threats. This pattern is consistent with the findings of Mermillod et al. (2010).

Surprisingly, the identification rates across the conditions followed a different pattern. A $2 \times 2$ Living Status (living vs. nonliving) $\times$ Threat Status (threatening vs. nonthreatening) repeated measures ANOVA revealed a main effect of living status, such that participants were actually more likely to identify the nonliving stimuli, both threats $(M=.44, S D=.13)$ and nonthreats $(M=.44, S D=.11)$, than to identify the living stimuli, both threats $(M=.22, S D=.12)$ and nonthreats $(M=.19, S D=.09), F(1,59)=371.06, M S E=.01, p<.001$, $\eta_{\mathrm{p}}{ }^{2}=.86$. We observed no main effect of threat status, $F(1,59)=1.08, p=.30$, but the interaction approached significance, $F(1,59)=3.21, M S E=.01, p=.078, \eta_{\mathrm{p}}{ }^{2}=.05$, and was such that whereas participants were more likely to identify living threats than living nonthreats, they were not more likely to identify nonliving threats than nonliving nonthreats. The latter pattern is consistent with Mermillod et al. (2010), in that there was something special about living threats: Living threats were more identifiable than living nonthreats, but nonliving threats were not more identifiable than nonliving nonthreats. However, the finding that participants identified more nonliving than living stimuli seems to contrast with the findings of New et al. (2007), who found that when living things changed, the changes were detected better in a change-detection paradigm than when nonliving things changed. One contributing factor to the difference in our pattern of identification results might be that identifying a stimulus through noise is different from detecting a change. Another possibility is that our nonliving stimuli were more frequently encountered in participants' daily lives than our living stimuli. For example, coffee mugs and guns are commonly seen in daily life and in the media, whereas snakes and rabbits may be less commonly encountered on a daily basis. This may lead to something akin to a word frequency perceptual identification effect (e.g., McClelland \& Rumelhart, 1981), in which items more frequently encountered in daily life are more easily identified in hard-to-identify circumstances. It is also possible that the nonliving stimuli had sharper edges than the living stimuli, making it somewhat easier to identify these through the noise. Still, the fact that we found a threat advantage to identification only among living stimuli is itself consistent with the findings of Mermillod et al. (2010).

Because the likelihood of identification was not evenly distributed across the categories (nonliving stimuli had a higher probability of being identified from the noise than 
did living stimuli), we wanted to determine whether the relative identifiability of the stimuli and the familiarity ratings to the stimuli were related (in a search for evidence for potential item selection effects). Toward this end, we examined whether the familiarity ratings for the items that had been unidentified from their noise filters varied systematically as a function of their relative identifiability from their noise filters within the context of the experiment. This was possible because all of the stimuli used in Experiment 3 were identifiable, insofar as they had clear names associated with them (unlike in the nonfamous conditions of Exps. 1 and 2).

We segmented all of the 80 items from Experiment 3 into quartiles according to the average proportions of participants who successfully identified the items. The first quartile consisted of the 20 most-identified items (range $=.53-.97$ of participants identified those items); the second quartile contained the 20 next-most-identified items (range $=.25-.52$ ); the third quartile consisted of the 20 next-to-least-identified items (range $=.05-.23$ ); and the fourth quartile consisted of the 20 least-identified items (range $=.00-.05)$. The mean familiarity ratings for unidentified images did not vary systematically as a function of their relative identifiability by participants in the study. A one-way ANOVA performed on the mean familiarity ratings for unidentified images revealed no significant effect of identification quartile (first, second, third, or fourth), $F(3,76)=1.46, M S E=1.13, p=.23$. Familiarity ratings did not follow a linear pattern, as can be seen when comparing the top quartile $(M=2.98, S D=1.23)$ with the second quartile $(M=3.10, S D=0.80)$, the third $(M=3.64, S D=1.33)$, and the fourth $(M=3.25, S D=0.79)$. Although this does not completely rule out the possibility of item selection effects, this evidence does not suggest a relationship between relative identifiability and the familiarity ratings given to unidentified stimuli. Relative identifiability and familiarity ratings to unidentified stimuli appear to be unrelated.

We also examined whether it would make a difference if we did not conditionalize the familiarity ratings for unidentified stimuli on the postexperiment identifiability of the stimuli unoccluded by the noise mask. The means and the overall pattern were very similar to those for the conditionalized data: The familiarity ratings for unidentified stimuli were still higher for living threats $(M=3.75, S D=$ $1.03)$ than for living nonthreats $(M=3.29, S D=1.03), t(59)=$ 3.70, $S E=0.13, p<.001, d=0.48$, while no such difference was found when comparing nonliving threats $(M=3.23$, $S D=1.07)$ with nonliving nonthreats $(M=3.06, S D=$ $0.90), t(59)=1.17, S E=0.15, p=.25$. Also, the familiarity ratings for unidentified living threats were still higher than those for unidentified nonliving threats, $t(59)=4.17$, $S E=0.13, p<.001, d=0.54$.

\section{General discussion}

This study demonstrates that even when visual images are too occluded by a monochromatic noise filter to be consciously identified, people can still detect relative familiarity or novelty with the images, and can even detect, via familiarity ratings, whether or not an unidentified living image presents a potential threat.

From an evolutionary perspective, some may view it as adaptive to be able to at least obtain a general sense about a situation for which not enough information is available to allow conscious identification. This general sense may allow for a quick determination of whether a face is a friend or enemy if the face is hidden, seen in the dark, or only seen for a quick glance. It may also allow a determination of whether a scene's layout is familiar or novel when given a mere quick glance or through hazy visual conditions.

However, although it is inherently adaptive, this ability to have a general sense about an unidentifiable face or scene may actually be due to expertise. Many have noted that one of the key characteristics of expertise is the ability to rapidly recognize patterns or critical features of a situation, as when a chess expert instantly knows what the next move should be or an expert radiologist instantly determines that there is an abnormality present (see, e.g., Gobet \& Chassy, 2009, for a review). Such "expert-based intuition" has been argued to be an important factor in effective decision-making within organizations (Salas, Rosen, \& DiazGranados, 2010), and although the argument is controversial, some have indeed linked face processing with expertise (e.g., Gauthier \& Bukach, 2007; Wong, Palmeri, \& Gauthier, 2009). Thus, the ability of participants to detect greater familiarity with famous than with nonfamous unidentified faces might be an intuition-based phenomenon derived from expertise with faces. A similar argument could be made with the famousversus-nonfamous scenes used in the present study. With regard to what allows the intuitive sense that an unidentified image is familiar, one potential explanation is that famous faces and famous scenes are processed more fluently than nonfamous faces or scenes (e.g., Hertwig, Herzog, Schooler, $\&$ Reimer, 2008), even when the increase in fluency is not enough to allow identification to occur.

Though the expertise and fluency ideas may be able to explain the real-world RWI effect that we have shown in the present study with famous faces and famous scenes, these ideas have more difficulty explaining our finding from Experiment 3 that unidentified living threats received higher familiarity ratings than did either unidentified living nonthreats or unidentified nonliving threats. Especially in light of our finding that conscious ratings of how threatening the stimuli seemed (i.e., nonliving threats were actually consciously rated as more threatening than living threats), and our finding that nonliving stimuli were more identifiable 
from the noise than were living stimuli, it is not obvious why living threats (e.g., a snake) should have been processed more fluently or have drawn upon greater expertise than did nonliving threats (e.g., a gun) or living nonthreats (e.g., a bunny). However, given that the evolutionary relevance of being able to detect danger in the face of minimal perceptual information may particularly apply to living or animated things (Mermillod et al., 2010; New et al., 2007; VanArsdall et al., in press), our paradigm may be tapping a core adaptive cognitive process.

Mermillod et al. (2010) found that threats can be rapidly detected from images that contain only coarse information that is low in spatial frequency. In fact, the high-spatialfrequency advantage that is usually found for supraliminal stimuli disappears when threat stimuli are used, suggesting that people may be able to recognize threats on the basis of less perceptual information than they can with other types of stimuli. This threat advantage to processing appears to be limited to living threats. Although our dependent measure differed from that of Mermillod et al. (whereas they asked participants to judge the threat for supraliminal stimuli after fear conditioning, we only asked participants to judge familiarity, and for unidentified stimuli, and not after any fear conditioning), our results are consistent with theirs in that we only showed a familiarity-rating discrimination effect for living (or animate) things, not for nonliving (or inanimate) things. Thus, our study provides converging evidence from a novel paradigm that something may be special about living or animate things with regard to threat detection in the face of minimal perceptual information.

Furthermore, because we did not ask participants to judge the likelihood that something was a threat, but rather, asked them to judge familiarity, our results also suggest a link between threat detection and familiar-novel detection. It is possible that what enables threat detection among unidentified living things is also a sense of familiarity or intuition. If so, then familiarity, intuition, and threat detection for unidentified stimuli may reflect a core, adaptive memory process.

The core process that enables familiar-novel discrimination in the absence of identification may be familiarity. Many researchers of recognition memory have long argued that recognition can occur solely on the basis of a general sense of familiarity (e.g., Diana, Reder, Arndt, \& Park, 2006; Mandler, 1991, 2008; Ryals \& Cleary, 2012; Yonelinas, 2002), and some have argued that familiarity detection itself may have an emotional component, possibly involving arousal (e.g., Goldinger \& Hansen, 2005; Morris et al., 2008). Future research should examine whether the effects reported here involve an emotional component.

Interestingly, some aspects of our study suggest that the intuition-based familiarity process that may be at work in detecting unidentifiable living threats differs altogether from the type of conscious threat identification that occurs for unmasked, unfiltered stimuli. Although our unidentified living threats were rated as more familiar than our unidentified nonliving threats, as mentioned, the conscious ratings of threat that we collected to independently assess whether our threatening stimuli were indeed threatening (as compared to our nonthreatening stimuli) were in the opposite direction: Those ratings were significantly higher for nonliving than for living threats. This suggests that the intuitive sense that allowed for higher familiarity ratings to be given to unidentified living stimuli in our study is not what drives the more deliberate, conscious perception of threat that occurs when more information is available.

Though the process that most studies of recognition memory aim to study is that which produces familiarity in day-to-day situations, such studies tend to use list-learning paradigms, whereby participants usually discriminate between two sets of known stimuli: those that received an increase in familiarity through experimental presentation, and those that did not. Ours is the first study to show that the familiarity presumed to underlie RWI in list-learning approaches can operate in the more day-to-day-like experience of distinguishing the familiar from the novel in the face of minimal perceptual information. In showing RWI of known versus novel things, our results are somewhat reminiscent of the findings of Bolte and Goschke (2008), who showed that participants could recognize unidentified linedrawing fragments as coming from "coherent" objects (i.e., a fragment of an otherwise intact typewriter) as opposed to from "incoherent" objects (i.e., a fragment from a typewriter that had been scrambled). Bolte and Goschke argued that this ability stems from participants forming an intuitive Gestalt, whereby existing knowledge of the Gestalts that becomes automatically activated in response to an unidentified fragment contributes to a general intuition about the unidentified fragment.

What is the relationship between familiarity detection and intuition? Both intuition (e.g., Gobet \& Chassy, 2009; Salas et al., 2010) and familiarity (e.g., Mandler, 2008) are described as a general sense about a stimulus or situation, without conscious access to specifics. Thus, intuition and familiarity may be intertwined. The results from the present study may be due to an intuitive sense about the famous images despite their being unidentifiable. This idea is akin to relying on the recognition heuristic as a means of "fast and frugal" decision-making (Gigerenzer \& Goldstein, 1996; Goldstein \& Gigerenzer, 2002). In particular, when only minimal information is available, people might need to rely on only familiarity, or only an intuitive sense about whether something is known or novel, to make a rapid decision. This may help people make decisions in the face of minimal information (Dudey \& Todd, 2002). 
Future research should further investigate the potential link between familiarity detection and intuition. Future research should also investigate whether the familiar-novel discrimination that occurs for unidentified faces and scenes has the same basis as the threat detection shown via familiarity ratings for unidentified living threats. It is possible that these different types of detection can be dissociated, which might help to shed light on the extent to which the RWI effects shown in the present study each reflect a domainspecific or domain-general adaptation. Also, in light of some previous work on the ability of participants to exhibit abovechance recognition unconsciously (e.g., Cleary, 2012; Voss \& Paller, 2009), future work should aim to examine the extent to which the RWI effect reported here results from conscious versus unconscious recognition processes.

Finally, the present paradigm may be of use for investigating applied questions, such as how to effectively design camouflage (Qi, Xu-Liang, \& Chao, 2011) or the opposite, how to train individuals to effectively break camouflage in order to detect threats (Chen \& Hegdé, 2012)

Given that people can still have an intuitive sense about a situation in the absence of conscious identification of what is driving that intuitive sense, it may be important to understand what types of masks are effective at eliminating the RWI effect in familiar-novel discrimination. For instance, if a type of mask or camouflage is only effective at reducing conscious identification itself, but not the intuitive sense about the situation, it may not be the most effective means of masking a situation.

Author note This project was supported by National Science Foundation Grant No. 0638486 to A.M.C.

\section{References}

Arndt, J., Lee, K., \& Flora, D. B. (2008). Recognition without identification for words, pseudowords and nonwords. Journal of Memory and Language, 59, 346-360.

Bolte, A., \& Goschke, T. (2008). Intuition in the context of object perception: Intuitive gestalt judgments rest on the unconscious activation of semantic representations. Cognition, $108,608-616$

Chen, X., \& Hegdé, J. (2012). Learning to break camouflage by learning the background. Psychological Science, 23, 1395-1403.

Cleary, A. M. (2011). Face recognition without identification. In P. M. Corcoran (Ed.), Reviews, refinements and new ideas in face recognition (pp. 317-328). Rijeka, Croatia: InTech.

Cleary, A. M. (2012). On the contribution of unconscious processes to recognition memory. Cognitive Neuroscience, 3, 210-211.

Cleary, A. M., \& Greene, R. L. (2000). Recognition without identification. Journal of Experimental Psychology: Learning, Memory, and Cognition, 26, 1063-1069.

Cleary, A. M., \& Greene, R. L. (2001). Memory for unidentified items: Evidence for the use of letter information in familiarity processes. Memory and Cognition, 29, 540-545. doi:10.3758/BF03196405
Cleary, A. M., \& Greene, R. L. (2005). Recognition without perceptual identification: A measure of familiarity? Quarterly Journal of Experimental Psychology, 58A, 1143-1152.

Cleary, A. M., Langley, M. M., \& Seiler, K. R. (2004). Recognition without picture identification: Geons as components of the pictorial memory trace. Psychonomic Bulletin and Review, 11, 903-908. doi:10.3758/BF03196719

Cleary, A. M. (2008). Recognition memory, familiarity, and déjà vu experiences. Current Directions in Psychological Science, 17, 353-357.

Cleary, A. M., \& Reyes, N. L. (2009). Scene recognition without identification. Acta Psychologica, 131, 53-62. doi:10.1016/ j.actpsy.2009.02.006

Cleary, A. M., \& Specker, L. E. (2007). Recognition without face identification. Memory and Cognition, 35, 1610-1619.

Cleary, A. M., Winfield, M. M., \& Kostic, B. (2007). Auditory recognition without identification. Memory and Cognition, 35, 18691877. doi:10.3758/BF03192921

Diana, R. A., Reder, L. M., Arndt, J., \& Park, H. (2006). Models of recognition: A review of arguments in favor of a dual-process account. Psychonomic Bulletin and Review, 13, 1-21. doi:10.3758/ BF03193807

Dudey, T., \& Todd, P. M. (2002). Making good decisions with minimal information: Simultaneous and sequential choice. Journal of Bioeconomics, 3, 195-215.

Gauthier, I., \& Bukach, C. (2007). Should we reject the expertise hypothesis? Cognition, 103, 322-330. doi:10.1016/j.cognition. 2006.05.003

Gigerenzer, G., \& Goldstein, D. G. (1996). Reasoning the fast and frugal way: Models of bounded rationality. Psychological Review, 103, 650-669. doi:10.1037/0033-295X.103.4.650

Gladwell, M. (2005). Blink: The power of thinking without thinking. New York, NY: Little, Brown.

Gobet, F., \& Chassy, P. (2009). Expertise and intuition: A tale of three theories. Minds and Machines, 19, 151-180.

Goldinger, S. D., \& Hansen, W. A. (2005). Remembering by the seat of your pants. Psychological Science, 16, 525-529.

Goldstein, D. G., \& Gigerenzer, G. (2002). Models of ecological rationality: The recognition heuristic. Psychological Review, 109, 75-90. doi:10.1037/0033-295X.109.1.75

Hertwig, R., Herzog, S. M., Schooler, L. J., \& Reimer, T. (2008). Fluency heuristic: A model of how the mind exploits a byproduct of information retrieval. Journal of Experimental Psychology: Learning, Memory, and Cognition, 34, 1191-1206. doi:10.1037/a0013025

Kostic, B., \& Cleary, A. M. (2009). Song recognition without identification: When people cannot "name that tune" but can recognize it as familiar. Journal of Experimental Psychology. General, 138, 146-159. doi:10.1037/a0014584

Lang, P. J., Bradley, M. M., \& Cuthbert, B. N. (2005). International Affective Picture System (IAPS): Affective ratings of picture and instruction manual (Technical Report A-6). Gainesville, FL: University of Florida, Center for Research in Psychophysiology.

Langley, M. M., Cleary, A. M., Woods, J., \& Kostic, B. (2008). Picture recognition without picture identification: A method for assessing the role of perceptual information in familiarity-based picture recognition. Acta Psychologica, 127, 103-113.

Larsson, S. (2008). The girl with the dragon tattoo (R. Keeland, Trans.). New York, NY: Knopf.

Loftus, G. R., \& Masson, M. E. J. (1994). Using confidence intervals in within-subject designs. Psychonomic Bulletin and Review, 1, 476-490. doi:10.3758/BF03210951

Mandler, G. (1991). Your face looks familiar but I can't remember your name: A review of dual process theory. In W. E. Hockley \& S. Lewandowsky (Eds.), Relating theory and data: Essays on human memory in honor of Bennett B. Murdock (pp. 207-225). Hillsdale, NJ: Erlbaum. 
Mandler, G. (2008). Familiarity breeds attempts: A critical review of dual-process theories of recognition. Perspectives on Psychological Science, 3, 390-399. doi:10.1111/j.17456924.2008.00087.x

McClelland, J. L., \& Rumelhart, D. E. (1981). An interactive activation model of context effects in letter perception: I. An account of basic findings. Psychological Review, 88, 375-407. doi:10.1037/ 0033-295X.88.5.375

Mermillod, M., Droit-Volet, S., Devaux, D., Schaefer, A., \& Vermeulen, N. (2010). Are coarse scales sufficient for fast detection of visual threat? Psychological Science, 21, 14291437.

Morris, A. L., Cleary, A. M., \& Still, M. S. (2008). The role of autonomic arousal in feelings of familiarity. Consciousness and Cognition, 17, 1378-1385.

New, J., Cosmides, L., \& Tooby, J. (2007). Category-specific attention for animals reflects ancestral priorities, not expertise. Proceedings of the National Academy of Sciences, 104, 16598-16603. doi:10.1073/pnas.0703913104

Peynircioğlu, Z. F. (1990). A feeling-of-recognition without identification. Journal of Memory and Language, 29, 493-500. doi:10.1016/0749-596X(90)90068-B

Qi, J., Xu-Liang, L., \& Chao, W. (2011). Evaluation of engineering camouflage effectiveness based on human visual attention mechanisms. In Proceedings of the 2011 IEEE International Conference on E-Business and E-Government (ICEE), 1-4. doi:10.1109/ICEBEG.2011.5881935

Ryals, A. J., \& Cleary, A. M. (2012). The recognition without cued recall phenomenon: Support for a feature-matching theory over a partial recollection account. Journal of Memory and Language, 66, 747-762.

Salas, E., Rosen, M. A., \& DiazGranados, D. (2010). Expertise-based intuition and decision making in organizations. Journal of Management, 36, 941-973.

VanArsdall, J. E., Nairne, J. S., Pandeirada, J. N. S., \& Blunt, J. R. (in press). Adaptive memory: Animacy processing produces mnemonic advantages. Experimental Psychology. doi:10.1027/ 1618-3169/a000186

Voss, J. L., \& Paller, K. A. (2009). An electrophysiological signature of unconscious recognition memory. Nature Neuroscience, 12, 349-355.

Wong, A. C. N., Palmeri, T. J., \& Gauthier, I. (2009). Conditions for facelike expertise with objects: Becoming a Ziggerin expert-but which type? Psychological Science, 20, 1108-1117. doi:10.1111/ j.1467-9280.2009.02430.x

Yonelinas, A. P. (2002). The nature of recollection and familiarity: A review of 30 years of research. Journal of Memory and Language, 46, 441-517. doi:10.1006/jmla.2002.2864 